\section{Comprehensive Assessment of Left Ventricular Wall Motion Abnormalities in Coronary Artery Disease Using Cardiac Magnetic Resonance}

\section{Ion Codreanu ${ }^{1,2 *}$, Tammy J Pegg ${ }^{3}$, Joseph B Selvanayagam ${ }^{4}$, Matthew D Robson ${ }^{3}$, Oliver J Rider ${ }^{3}$, Constantin A Dasanu ${ }^{5}$, Bernd A Jung ${ }^{6,7}$, David P Taggart ${ }^{8}$, Natalia Rotaru ${ }^{2}$, Kieran Clarke $^{1}$ and Cameron J Holloway ${ }^{1,3,9}$}

'Department of Physiology, Anatomy and Genetics, University of Oxford, Oxford, UK

${ }^{2}$ Department of Radiology, Medpark International Hospital, Nicolae Testemitanu State University of Medicine and Pharmacy, Chișinău, Moldova ${ }^{3}$ Radcliffe Department of Medicine, University of Oxford Centre for Clinical Magnetic Resonance Research, Oxford, UK

${ }^{4}$ Department of Cardiovascular Medicine, Flinders University, Bedford Park, SA, Australia

${ }^{5}$ Department of Medicine, University of Connecticut, Farmington, CT, USA ${ }^{6}$ Department of Diagnostic Radiology, Medical Physics, University Hospital, Freiburg, Germany

${ }^{7}$ Institute of Diagnostic, Interventional and Pediatric Radiology, University Hospital, Bern, Switzerland

${ }^{8}$ Department of Cardiothoracic Surgery, John Radcliffe Hospital, Oxford, UK

${ }^{9}$ Department of Cardiology, St Vincent's Hospital, Sydney, New South Wales, Australia

\begin{abstract}
Objective: To obtain new details of three-dimensional Left Ventricular (LV) wall motion in patients with Coronary Artery Disease (CAD) using cardiac magnetic resonance navigator gated high temporal resolution tissue phase mapping.

Methods: LV motion patterns, myocardial velocities and time-to-peak values for radial, rotational and longitudinal motion were obtained in 19 patients with triple vessel CAD and in a group
\end{abstract}

*Corresponding author: Ion Codreanu, Department of Physiology, Anatomy \& Genetics, University of Oxford, Sherrington Building, Parks Road Oxford, OX1 3PT, UK, Tel: +44 1865282246; Fax: +44 1865282272; E-mail: codrion@yahoo.com

Citation: Codreanu I, Pegg TJ, Selvanayagam JB, Robson MD, Rider OJ, et al. (2015) Comprehensive Assessment of Left Ventricular Wall Motion Abnormalities in Coronary Artery Disease Using Cardiac Magnetic Resonance. J Cardiol Stud Res 2: 006.

Received: February 09, 2015; Accepted: March 17, 2015; Published: March 31, 2015

Copyright: ( 2015 Codreanu I, et al. This is an open-access article distributed under the terms of the Creative Commons Attribution License, which permits unrestricted use, distribution, and reproduction in any medium, provided the original author and source are credited. of 9 age-matched healthy subjects. Radial, circumferential and longitudinal velocity curves were recorded separately for 16 ventricular segments. Ventricular torsion rate and longitudinal strain rate were also derived for each group.

Results: Markedly reduced peak diastolic longitudinal velocities $(-7.9 \pm 0.6$ versus $-14.1 \pm 1.1 \mathrm{~cm} / \mathrm{s})$ and peak diastolic longitudinal strain rates $\left(1.1 \pm 0.2\right.$ versus $\left.2.7 \pm 0.1 \mathrm{~s}^{-1}\right)$ were the most prominent findings in CAD. Patients with $C A D$ also demonstrated lower peak radial velocities, the difference increasing towards the LV apex (peak systolic values of $3.1 \pm 0.2$ versus $3.5 \pm 0.2 \mathrm{~cm} / \mathrm{s}$ at the LV base and $2.4 \pm 0.2$ versus $3.5 \pm 0.2 \mathrm{~cm} / \mathrm{s}$ at the LV apex), with sub-endocardial myocardium being affected to a greater extent. Prominent changes in rotational velocities and an altered pattern of ventricular rotation were also noted in CAD patients.

Conclusion: The study revealed complex alterations in radial, rotational and longitudinal LV wall motion in patients with CAD. Peak diastolic longitudinal velocity at the LV base and peak diastolic longitudinal strain rate seem particularly useful for detecting early changes in LV dysfunction associated to CAD. Evaluation of the pathophysiological interplays and underlying mechanisms of these changes require further studying and can provide new insights into understanding the pathophysiology of CAD.

Keywords: Cardiovascular magnetic resonance; Coronary artery disease; Myocardial velocities; Tissue phase mapping; Ventricular wall motion

\section{Abbreviations}

CAD: Coronary Artery Disease

LV: Left Ventricular

MR: Magnetic Resonance

TPM: Tissue Phase Mapping

\section{Introduction}

Subtle changes in Left Ventricular (LV) wall motion patterns have diagnostic and prognostic value in patients with Coronary Artery Disease (CAD) [1-10], and can precede chest pain and electrocardiographic changes [11,12]. Additionally, ventricular strain rate measurements can improve assessment of myocardial viability [13]. For instance, combined assessment of subendocardial contractile impairment and post-systolic shortening (defined as the peak strain after end systole) was demonstrated to be useful in identifying hypoperfused myocardium in the absence of visual wall motion abnormalities [11]. Quantitative measurement of myocardium strain and myocardium strain rate showed promising results in the evaluation of infarct size in patients with anterior ST-Elevation Myocardial Infarction (STEMI) [14]. Segmental strain and strain rate measurements in longitudinal, radial and circumferential directions have also been reported useful for predicting the presence of coronary collateral circulation in the ischemic myocardium [15].

Cardiac Magnetic Resonance (CMR) provides a comprehensive evaluation for patients with $\mathrm{CAD}$, including cardiac anatomy, function, viability and myocardial perfusion. Long and short axis cine images provide qualitative assessment of global and regional wall motion abnormalities, though the value of using CMR for comprehensive assessment of $3 \mathrm{D}$ regionality in CAD is not well established. Previously, we used the CMR navigator gated, high temporal resolution Tissue Phase Mapping (TPM) for investigating LV remodeling following Coronary Artery By-pass Graft surgery 
(CABG) [16]. Using the same methodology, subsequently, we investigated age related changes in regional and global myocardial wall motion in healthy individuals without cardiac disease [17]. In the current study, we aimed to compare three-dimensional LV wall motion in patients with CAD with similar data obtained in their age-matched healthy controls.

\section{Methods and Materials}

\section{Study population}

Nineteen patients $(65.7 \pm 7.4$ years old $)$ with triple vessel CAD documented by invasive coronary angiography were included into the study. All patients had proven coronary lesions warranting CABG. The lesions involved at least $70 \%$ obstruction in the left anterior descending artery $(n=16)$, circumflex artery $(n=12)$ or right coronary artery $(\mathrm{n}=15)$. Apart from this, 14 patients demonstrated stenotic lesions involving their left main segment (at least 50\% obstruction). Their pre-and postoperative characteristics, the affected coronary vessels as well as the number of applied coronary grafts have been reported in detail as part of a separate study investigating LV remodeling following CABG [16].

The majority of patients had risk factors for CAD, including hypercholesterolaemia (15 patients), hypertension (12 patients), smoking (9 patients), diabetes mellitus (8 patients), and peripheral vascular disease (6 patients). All patients were receiving medical therapy, including lipid lowering medication (19 patients), ACE inhibitors (17 patients), aspirin (17 patients), beta blockers (15 patients) and diuretics (7 patients). In this group with CAD, Left Ventricular (LV) ejection fraction averaged $40.0 \pm 2.5 \%$ (mean $\pm \mathrm{SD}$ ), cardiac output $5.6 \pm 0.3 \mathrm{l} / \mathrm{min}$, cardiac index $2.7 \pm$ $1.2 \mathrm{l} / \mathrm{min} / \mathrm{m}^{2}$, end systolic volume $151.5 \pm 15.7 \mathrm{ml}$, end diastolic volume $244.2 \pm 17.2 \mathrm{ml}, \mathrm{LV}$ mass $162.0 \pm 13.9 \mathrm{~g}, \mathrm{LV}$ mass index $80.1 \pm 4.9 \mathrm{~g} / \mathrm{m}^{2}$. Since CABG is expected to add a variety of confounding factors that affect LV wall motion, only the pre-CABG data were used in the current study.

A group of 9 age-matched healthy controls ( $66 \pm 7$ years old) were also studied. All healthy volunteers were non-smokers and underwent a medical assessment prior to acceptance in the study to exclude a history of cardiovascular and metabolic disease. Their baseline data have also been used in a separate study to investigate age-related changes in LV wall motion in healthy individuals [17]. The study was performed according to the principles of the declaration of Helsinki and was approved by a local clinical research ethics committee. Each subject provided written informed consent.

\section{Cardiac MR scans}

The cardiac MR scans were performed using a 1.5-T Siemens Sonata clinical scanner (Siemens Healthcare, Erlangen, Germany). Pilot images, followed by horizontal and vertical long axis cine images, were acquired using a steady-state Free Precession (TrueFISP) pulse sequence. Cine images for navigator-gated high temporal resolution phase contrast velocity mapping were acquired using a black blood segmented k-space spoiled gradient echo sequence $[18,19]$ with a temporal resolution of $13.8 \mathrm{~ms}$, with prospective ECG-gating and advanced respiratory navigator gating $\left(\mathrm{TR}=13.8 \mathrm{~ms}\right.$, flip angle $=15^{\circ}$, bandwidth $=650 \mathrm{~Hz} /$ pixel, FOV $=400 \times 300 \mathrm{~mm}$, matrix $=256 \times 96$ ), as previously described [16,20-23]. Three equidistant short axis images were acquired for the LV base, mid-ventricle and apex. The basal slice was positioned parallel to the base of the heart and distal to the LV outflow tract. Basal, mid-ventricular and apical slices were positioned 15-20 $\mathrm{mm}$ apart, depending on the heart size. With cardiac and respiratory gating, each short axis acquisition took approximately 3-5 min, with an average of 60-70 phases per cardiac cycle. Velocity encoding was performed by including a phase image with no velocity encoding followed by images with a bipolar gradient in the read, phase or slice direction after each radiofrequency pulse to the otherwise identical sequence (Velocity-Encoded gradient echo imaging (VENC) in-plane $=20 \mathrm{~cm} / \mathrm{s}$, VENC through-plane $=30 \mathrm{~cm} / \mathrm{s}$ ). Post processing was performed in the standard fashion of subtracting the phase from the image with no velocity encoding, followed by conversion of the phase data into velocity maps. Separate measurements were performed for the inner (endocardial) and outer (epicardial) layers. Transmural peak velocities were inferred automatically as averaged peak values of the inner and outer layers. The duration of the cardiac cycle was determined by the R-R interval on the electrocardiogram, with end systole defined as the smallest LV cavity. The cardiac phases were subsequently calculated and normalized for the entire group based on their average duration during a cardiac cycle $[16,20,23]$.

Tissue Phase Mapping (TPM) analysis was performed using customised software (MATLAB ${ }^{\circledR}$, v. 6.5; MathWorks ${ }^{\circledR}$, Natick, MA). The endocardial and epicardial borders were manually contoured for the base, mid ventricle and apex for each phase of the cardiac cycle. For the TPM analysis, the left ventricle was divided into 16 segments ( 6 basal, 6 middle and 4 apical) according to the American Heart Association model [24]. In-plane velocities were transformed into an internal polar coordinate system positioned at the centre of the mass of the left ventricle. Global ventricular velocity time courses for radial, circumferential and longitudinal motion were calculated for each group by averaging over the entire segmentation mask. In addition, graphical representations of all ventricular velocities during a cardiac cycle for individual LV segments were obtained. Global ventricular torsion and strain rates were also determined.

\section{Statistical analysis}

MATLAB files containing TPM data were converted and generated into Excel $^{\mathrm{TM}}$ (Microsoft Corporation, Redmond, WA) files for statistical analysis. Subsequently, all data were analysed using SPSS $^{\circledast}$ v. 16.0 (SPSS Inc. Chicago, IL) and MedCalc statistical software, v. 12.5.0 (MedCalc Software, Ostend, Belgium). The variables were tested for normal distribution and, after the assumption was met, a pooled t-test was used to compare the values between the two groups. Otherwise a non-parametric (Mann-Whitney) test was used. A $p$-value of less than 0.05 was considered significant.

\section{Results}

\section{Radial motion}

The peak systolic and diastolic radial velocities at the LV base, mid-ventricle and apex and their corresponding peak times are presented in table 1. Graphical representation of radial velocities at the LV base, mid-ventricle and apex during different phases of the cardiac cycle is shown in figure 1 (a - peak systolic radial velocity, b - peak diastolic radial velocity). Positive values reflect LV segmental motion towards the centre of the ventricle, whilst negative values reflect an outward motion. In patients with CAD, the radial component of ventricular contraction was affected most prominently at the LV apex (peak systolic transmural velocity of $2.4 \mathrm{~cm} / \mathrm{s}$ in CAD, versus $3.5 \mathrm{~cm} / \mathrm{s}$ in healthy controls; $p$-value $<0.01)$. The contractility of the LV base 
Citation: Codreanu I, Pegg TJ, Selvanayagam JB, Robson MD, Rider OJ, et al. (2015) Comprehensive Assessment of Left Ventricular Wall Motion Abnormalities in Coronary Artery Disease Using Cardiac Magnetic Resonance. J Cardiol Stud Res 2: 006.

- Page 3 of $11 \bullet$

\begin{tabular}{|c|c|c|c|c|c|c|c|}
\hline \multicolumn{2}{|c|}{$\begin{array}{l}\text { LV level and myocardial } \\
\text { layer }\end{array}$} & CAD patients & Healthy controls & $p$-value & CAD patients & Healthy controls & $p$-value \\
\hline \multicolumn{5}{|c|}{ Peak systolic radial velocity $(\mathrm{cm} / \mathrm{s})$} & \multicolumn{3}{|c|}{ Time to peak values (\% of ES) } \\
\hline \multirow{3}{*}{ LV base } & epicardial & $2.8 \pm 0.2$ & $3.1 \pm 0.2$ & 0.11 & $38.5 \pm 2.0$ & $42.7 \pm 2.6$ & 0.23 \\
\hline & transmural & $3.1 \pm 0.2$ & $3.5 \pm 0.2$ & 0.07 & $38.2 \pm 2.1$ & $42.6 \pm 2.6$ & 0.22 \\
\hline & endocardial & $3.4 \pm 0.2$ & $4.0 \pm 0.2$ & 0.06 & $38.1 \pm 1.9$ & $43.6 \pm 2.6$ & 0.11 \\
\hline \multirow{3}{*}{ Mid-ventricle } & epicardial & $2.7 \pm 0.2$ & $3.2 \pm 0.3$ & 0.11 & $36.8 \pm 1.8$ & $39.7 \pm 1.4$ & 0.33 \\
\hline & transmural & $3.0 \pm 0.2$ & $3.5 \pm 0.3$ & 0.06 & $39.6 \pm 2.3$ & $42.7 \pm 2.1$ & 0.42 \\
\hline & endocardial & $3.3 \pm 0.2$ & $4.0 \pm 0.3$ & 0.04 & $41.1 \pm 2.1$ & $44.7 \pm 1.5$ & 0.28 \\
\hline \multirow{3}{*}{ LV apex } & epicardial & $2.4 \pm 0.2$ & $3.2 \pm 0.2$ & $<0.01$ & $38.8 \pm 1.9$ & $38.6 \pm 1.9$ & 0.97 \\
\hline & transmural & $2.4 \pm 0.2$ & $3.5 \pm 0.2$ & $<0.01$ & $39.7 \pm 1.9$ & $39.1 \pm 1.7$ & 0.86 \\
\hline & endocardial & $2.5 \pm 0.2$ & $3.8 \pm 0.2$ & $<0.01$ & $44.3 \pm 1.8$ & $40.1 \pm 1.4$ & 0.17 \\
\hline \multicolumn{5}{|c|}{ Peak diastolic radial velocity $(\mathrm{cm} / \mathrm{s})$} & \multicolumn{3}{|c|}{ Time to peak values (\% of ED) } \\
\hline \multirow{3}{*}{ LV base } & epicardial & $-3.4 \pm 0.3$ & $-4.4 \pm 0.4$ & 0.03 & $18.9 \pm 0.9$ & $19.1 \pm 1.8$ & 0.90 \\
\hline & transmural & $-3.8 \pm 0.4$ & $-5.5 \pm 0.4$ & $<0.01$ & $19.0 \pm 0.9$ & $18.8 \pm 1.9$ & 0.92 \\
\hline & endocardial & $-4.3 \pm 0.4$ & $-6.6 \pm 0.5$ & $<0.01$ & $19.1 \pm 1.0$ & $18.8 \pm 1.7$ & 0.85 \\
\hline \multirow{3}{*}{ Mid-ventricle } & epicardial & $-3.0 \pm 0.3$ & $-4.3 \pm 0.2$ & $<0.01$ & $23.8 \pm 1.2$ & $23.2 \pm 1.2$ & 0.78 \\
\hline & transmural & $-3.3 \pm 0.3$ & $-5.2 \pm 0.2$ & $<0.01$ & $24.3 \pm 1.4$ & $23.5 \pm 1.1$ & 0.76 \\
\hline & endocardial & $-3.6 \pm 0.3$ & $-6.1 \pm 0.3$ & $<0.01$ & $24.1 \pm 1.4$ & $23.5 \pm 1.1$ & 0.80 \\
\hline \multirow{3}{*}{ LV apex } & epicardial & $-3.1 \pm 0.3$ & $-4.2 \pm 0.3$ & 0.01 & $24.1 \pm 1.3$ & $27.4 \pm 1.4$ & 0.15 \\
\hline & transmural & $-3.3 \pm 0.3$ & $-4.7 \pm 0.3$ & $<0.01$ & $24.3 \pm 1.2$ & $27.4 \pm 1.4$ & 0.16 \\
\hline & endocardial & $-3.5 \pm 0.3$ & $-5.2 \pm 0.4$ & $<0.01$ & $24.3 \pm 1.2$ & $27.4 \pm 1.4$ & 0.16 \\
\hline
\end{tabular}

Table 1: Peak radial velocities and time to peak values in patients with CAD and healthy controls*

* All values are presented as mean \pm SEM

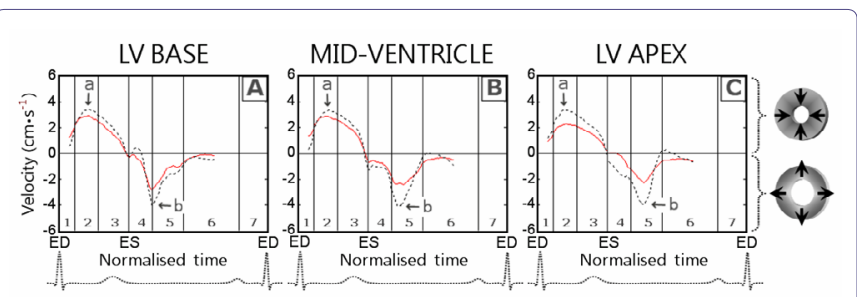

Figure 1: Radial velocity graphs at the LV base (panel A), mid-ventricle (panel B) and LV apex (panel C).

Positive values reflect LV motion towards the centre of the ventricle, whilst negative values reflect an outward motion. Solid line - patients with CAD; Dotted line - healthy controls; Arrows point towards peak systolic (a) and peak diastolic (b) radial velocities; Cardiac cycle phases: 1 - isovolumetric contraction; 2 - rapid ejection; 3 - reduced ejection; 4 - isovolumetric relaxation; 5 - rapid filling; 6 - diastasis; and 7 - atrial systole; ED: End Diastole; ES: End Systole

was also affected, even though to a lower degree $(p$-value $=0.07)$. The most affected myocardial layer in patients with CAD was the inner/endocardial layer (Table 1). Whilst LV contraction in CAD was affected more towards the apex (a 11.4\% decrease in peak systolic velocity at the LV base versus a $31.4 \%$ decrease at the LV apex), the ventricular expansion in diastole was affected to a similar degree at all LV levels (a 30.9\% decrease in peak diastolic transmural velocity at the LV base versus a $29.8 \%$ decrease at the LV apex). Furthermore, the difference in peak diastolic radial velocities was statistically significant at all ventricular levels (Table 1 , all $p$-values $<0.05)$. The time to peak systolic and diastolic radial velocities, however, showed no difference between the control and CAD groups.

The radial velocities for individual ventricular segments at the LV base, mid-ventricle and LV apex are displayed in figure 2,3 and 4 respectively. The difference between radial motion of apical segments in the two groups appeared particularly prominent on segmental velocity curves. Segmental graphs also showed that anterior (i.e., segments 1 and 7) and inferior segments (i.e., segments 4 and 10) were affected to a higher degree compared with septal and lateral segments (Figure 2 and 3).

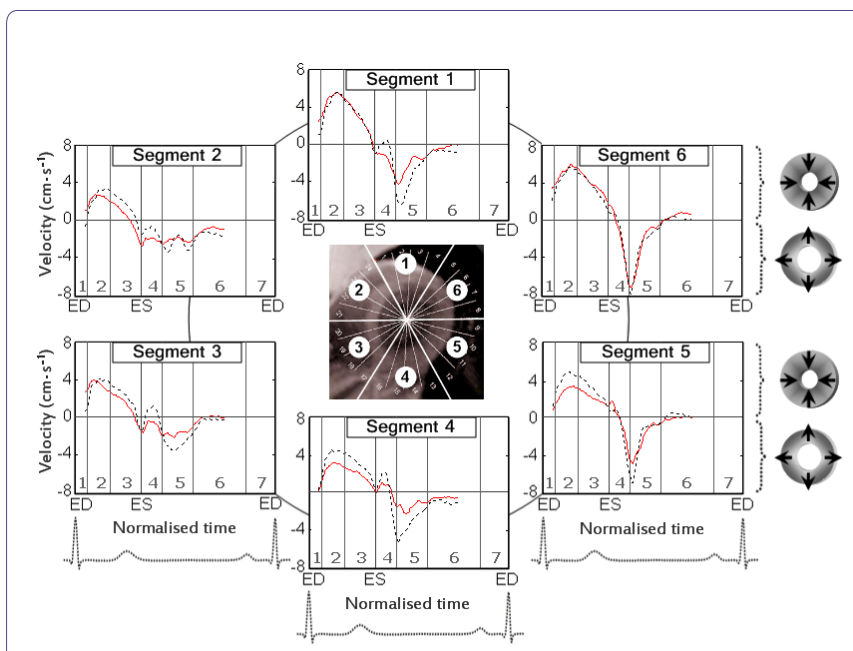

Figure 2: Radial velocity graphs for basal LV segments during a cardiac cycle. Solid line - patients with CAD; Dotted line - healthy controls; Segments: 1 - basal anterior segment; 2 - basal anteroseptal segment; 3 - basal inferoseptal segment; 4 - basal inferior segment; 5 - basal inferolateral segment; 6 - basal anterolateral segment; Cardiac cycle phases: 1 - isovolumetric contraction; 2 - rapid ejection; 3 - reduced ejection; 4 - isovolumetric relaxation; 5 - rapid filling; 6 - diastasis; and 7 - atrial systole; ED: End Diastole; ES: End Systole 


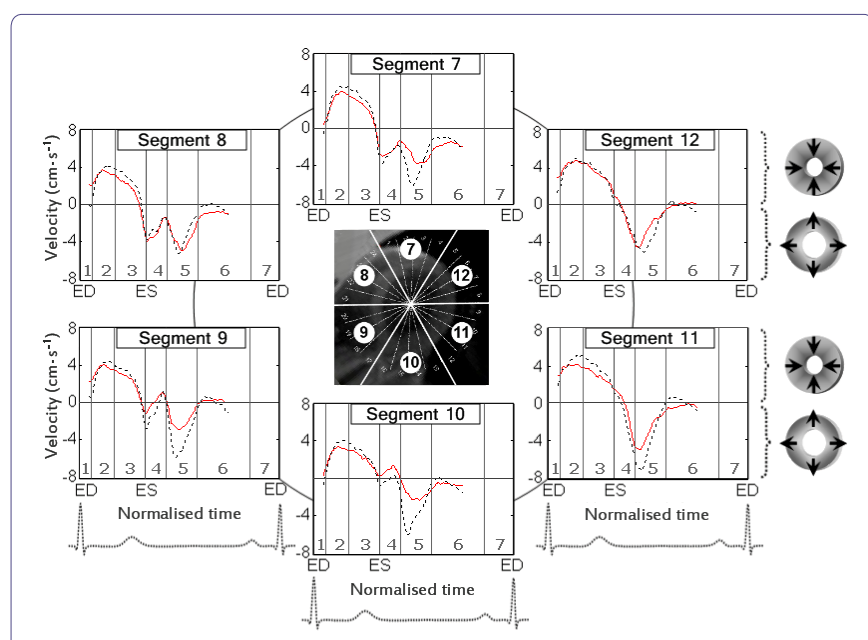

Figure 3: Radial velocity graphs for mid-ventricular segments during a cardiac cycle.

Solid line - patients with CAD; Dotted line - healthy controls; Segments: 7 - mid-anterior segment; 8 - mid-anteroseptal segment; 9 - mid-inferoseptal segment; 10 - mid-inferior segment; 11 - mid-inferolateral segment; 12 - mid-anterolateral segment; Cardiac cycle phases: 1 - isovolumetric contraction; 2 - rapid ejection; 3 - reduced ejection; 4 - isovolumetric relaxation; 5 - rapid filling; 6 - diastasis; 7 - atrial systole; ED: End Diastole; ES: End Systole

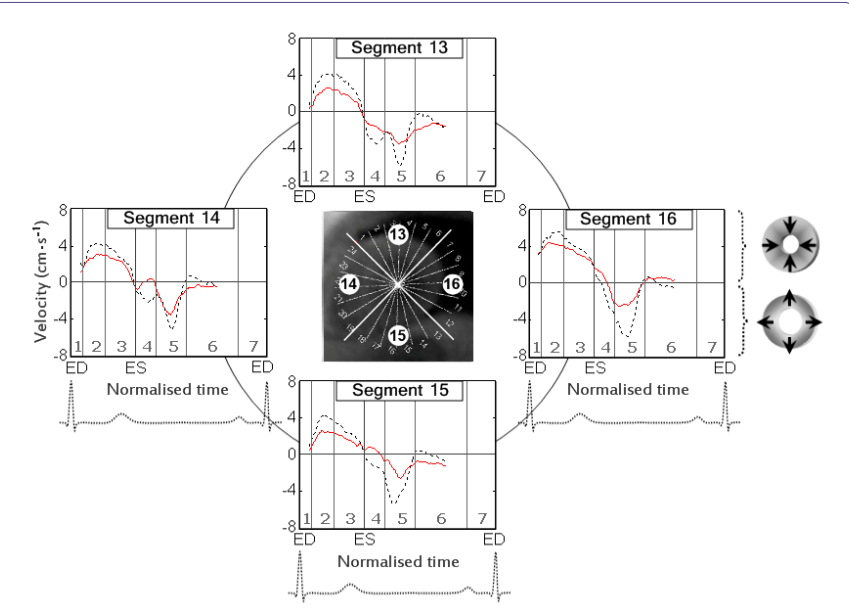

Figure 4: Radial velocity graphs for apical LV segments during a cardiac cycle.

Solid line - patients with CAD; Dotted line - healthy controls; Segments: 13 - apical anterior segment; 14 - apical septal segment; 15 - apical inferior segment; 16 - apical lateral segment; Cardiac cycle phases: 1 - isovolumetric contraction; 2 - rapid ejection; 3 - reduced ejection; 4 - isovolumetric relaxation; 5 - rapid filling; 6 - diastasis; and 7 - atrial systole; ED: End Diastole; ES: End Systole

\section{Rotational motion}

Peak clockwise and counter-clockwise circumferential velocities for both study groups are provided in table 2. Positive values reflect clockwise rotation and negative values reflect counter-clockwise rotation as viewed from the apex. The graphical representation of circumferential velocities at the LV base, mid-ventricle and apex is shown in figure 5. An altered pattern of ventricular untwisting was observed in patients with CAD, with much higher peak clockwise velocities at the LV apex (Figure 5, wave h). In healthy volunteers, the circumferential velocity graph at the LV apex showed an undulating pattern of alternating waves of counter-clockwise rotation (Figure 5, dotted line, arrows a, c and e) and waves of sudden deceleration or opposite rotations (arrows $\mathrm{b}, \mathrm{d}$ and $\mathrm{f}$ ). In patients with CAD, these waves of alternating rotational motion were less prominent (Figure 5, waves a-h, solid line). For instance, in CAD patients, after the initial counter-clockwise rotation of the entire left ventricle (Figure 5, arrow a), the velocity of the counter-clockwise rotation at the LV apex decreased continually during systole and was subsequently followed by a single wave of clockwise rotation in diastole (arrow $\mathrm{h}$ ).

Whilst in healthy volunteers, the LV apex at the end of systole was rotating in a clockwise direction (Figure 5, dashed line, arrow d), in CAD patients the apex continued its counter-clockwise rotation, with a prominent delay in rotational motion. In healthy controls, the recoil clockwise rotation of the LV apex started after repolarization and before the end of systole (Figure 5, dotted line, wave $\mathrm{d}$ becomes positive before the end systole), whilst in CAD patients it started much later, during the second part of isovolumetric relaxation. In diastole, the LV apex in healthy controls showed two separate waves of recoil clockwise rotation (Figure 5, dotted line, arrows $d$ and $\mathrm{f}$ ), alternating with opposing waves of counter-clockwise motion. In CAD patients, however, the recoil clockwise rotation of the LV apex started much later and continued as a single wave (Figure 5, solid line, arrow h). Thus, during the first half of rapid ventricular filling, the $\mathrm{LV}$ apex was rotating in a clockwise direction in CAD patients and in a counter-clockwise direction in healthy controls (Figure 5, dotted line is below zero during the first half of phase 5 , while solid line is well above zero during the entire phase 5). Of interest are also the higher peak velocities during the initial counter-clockwise rotation at the commencement of systole, obtained in CAD patients (Table 2). The difference was more pronounced at the LV apex $(4.5 \mathrm{~cm} / \mathrm{s}$ in CAD patients compared with $3.7 \mathrm{~cm} / \mathrm{s}$ in healthy controls), although it did not reach statistical significance in our study ( $p$-values between 0.07 and 0.2 ).

The time to peak counter-clockwise velocity at the LV apex and mid-ventricle in both control and CAD groups was represented by the initial counter-clockwise rotation of the entire ventricle (Figure 5 $(\mathrm{B}, \mathrm{C})$, arrow a), whilst at the LV base by the first wave of recoil counter-clockwise rotation of the $\mathrm{LV}$ base during untwisting (Figure 5A, arrow e).

The time to peak clockwise velocity at the LV base was represented in both groups by the clockwise rotation of the ventricular base during ejection (Figure 5A, arrow b). At the mid-ventricular level, the time to peak clockwise velocity in healthy controls was divided in accordance with two different waves of clockwise rotation located at $99.7 \%$ and $147.5 \%$ of end-systole (Figure 5B, arrows $\mathrm{d}$ and $\mathrm{f}$, dotted line). In different subjects, the time to peak clockwise velocity at the mid-ventricle was represented by any of these two peaks, which is also reflected in table 2). In CAD patients, the time to peak clockwise velocity at the mid-ventricular level was represented by a single prominent wave of clockwise rotation transmitted from the apex (Figure 5B, arrow h, solid line). At the LV apex, the time to peak clockwise velocity in healthy controls was represented by the first recoil wave of clockwise rotation (Figure 5C, arrow d, dotted line), whilst in CAD patients-by the delayed wave of apical untwisting with a prominent single peak of clockwise recoil motion (Figure 5C, arrow $\mathrm{h}$, solid line). 
Citation: Codreanu I, Pegg TJ, Selvanayagam JB, Robson MD, Rider OJ, et al. (2015) Comprehensive Assessment of Left Ventricular Wall Motion Abnormalities in Coronary Artery Disease Using Cardiac Magnetic Resonance. J Cardiol Stud Res 2: 006.

- Page 5 of $11 \bullet$

\begin{tabular}{|c|c|c|c|c|c|c|c|}
\hline \multicolumn{2}{|c|}{$\begin{array}{c}\text { LV level and myocardial } \\
\text { layer }\end{array}$} & CAD patients & Healthy controls & $p$-value & CAD patients & Healthy controls & $p$-value \\
\hline \multicolumn{5}{|c|}{ Peak clockwise velocity $(\mathrm{cm} / \mathrm{s})$} & \multicolumn{3}{|c|}{ Time to peak values (\% of ES) } \\
\hline \multirow{3}{*}{ LV base } & epicardial & $2.4 \pm 0.2$ & $2.6 \pm 0.3$ & 0.35 & $51.2 \pm 2.0$ & $47.5 \pm 1.1$ & 0.25 \\
\hline & transmural & $2.3 \pm 0.2$ & $2.5 \pm 0.3$ & 0.27 & $51.5 \pm 2.0$ & $47.5 \pm 1.1$ & 0.21 \\
\hline & endocardial & $2.2 \pm 0.2$ & $2.5 \pm 0.3$ & 0.24 & $51.2 \pm 1.8$ & $46.3 \pm 1.1$ & 0.10 \\
\hline \multirow{3}{*}{ Mid-ventricle } & epicardial & $2.9 \pm 0.4$ & $2.0 \pm 0.2$ & 0.09 & $137.5 \pm 2.3$ & $\begin{array}{l}101.2 \pm 0.6^{\dagger} \\
147.5 \pm 5.1^{\dagger}\end{array}$ & $<0.01$ \\
\hline & transmural & $2.7 \pm 0.4$ & $2.0 \pm 0.3$ & 0.14 & $137.0 \pm 2.2$ & $\begin{array}{c}99.7 \pm 2.0^{\dagger} \\
147.5 \pm 5.1^{+} \\
\end{array}$ & $<0.01$ \\
\hline & endocardial & $2.6 \pm 0.4$ & $2.2 \pm 0.3$ & 0.25 & $140.3 \pm 3.2$ & $\begin{array}{c}99.7 \pm 2.0^{\dagger} \\
147.5 \pm 5.1^{\dagger} \\
\end{array}$ & $<0.01$ \\
\hline \multirow{3}{*}{ LV apex } & epicardial & $4.0 \pm 0.5$ & $2.3 \pm 0.3$ & 0.02 & $137.1 \pm 2.5$ & $109.9 \pm 4.6$ & $<0.01$ \\
\hline & transmural & $3.8 \pm 0.5$ & $2.2 \pm 0.2$ & 0.02 & $137.6 \pm 2.5$ & $109.9 \pm 4.7$ & $<0.01$ \\
\hline & endocardial & $3.6 \pm 0.5$ & $2.2 \pm 0.2$ & 0.03 & $135.3 \pm 3.1$ & $111.4 \pm 5.1$ & $<0.01$ \\
\hline \multicolumn{5}{|c|}{ Peak counterclockwise velocity $(\mathrm{cm} / \mathrm{s})$} & \multicolumn{3}{|c|}{ Time to peak values (\% of ES) } \\
\hline \multirow{3}{*}{ LV base } & epicardial & $-3.7 \pm 0.4$ & $-3.3 \pm 0.4$ & 0.25 & $117.7 \pm 2.1$ & $117.6 \pm 1.5$ & 0.98 \\
\hline & transmural & $-3.5 \pm 0.4$ & $-3.3 \pm 0.4$ & 0.34 & $119.4 \pm 1.9$ & $118.1 \pm 1.5$ & 0.70 \\
\hline & endocardial & $-3.4 \pm 0.4$ & $-3.4 \pm 0.3$ & 0.48 & $119.0 \pm 2.0$ & $118.6 \pm 1.7$ & 0.98 \\
\hline \multirow{3}{*}{ Mid-ventricle } & epicardial & $-3.8 \pm 0.4$ & $-3.5 \pm 0.4$ & 0.34 & $27.8 \pm 1.6$ & $25.3 \pm 1.9$ & 0.39 \\
\hline & transmural & $-3.6 \pm 0.4$ & $-3.5 \pm 0.3$ & 0.44 & $27.2 \pm 1.6$ & $25.3 \pm 1.9$ & 0.51 \\
\hline & endocardial & $-3.4 \pm 0.4$ & $-3.5 \pm 0.3$ & 0.46 & $27.6 \pm 1.4$ & $25.3 \pm 1.9$ & 0.41 \\
\hline \multirow{3}{*}{ LV apex } & epicardial & $-4.7 \pm 0.4$ & $-3.8 \pm 0.4$ & 0.07 & $29.5 \pm 1.3$ & $26.2 \pm 1.1$ & 0.13 \\
\hline & transmural & $-4.5 \pm 0.4$ & $-3.7 \pm 0.4$ & 0.13 & $29.8 \pm 1.3$ & $26.2 \pm 1.1$ & 0.11 \\
\hline & endocardial & $-4.2 \pm 0.4$ & $-3.7 \pm 0.4$ & 0.20 & $29.6 \pm 1.3$ & $26.7 \pm 1.4$ & 0.24 \\
\hline
\end{tabular}

Table 2: Peak circumferential velocities and time to peak values in patients with $C A D$ and healthy controls*.

${ }^{*}$ All values are presented as mean \pm SEM

${ }^{\dagger}$ In healthy controls, the time to peak clockwise velocity at mid-ventricular level was represented by two separate waves in different subjects (Figure 5B, dotted line, waves $d$ and $f$ )

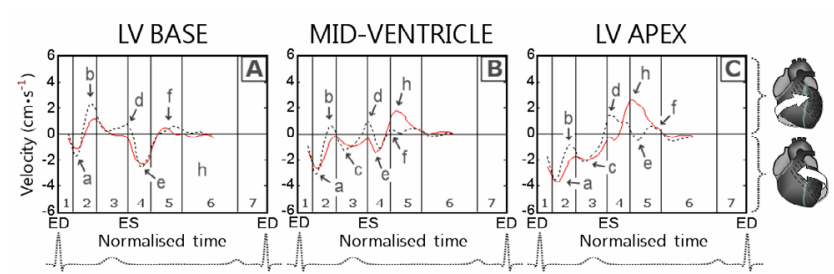

Figure 5: Circumferential velocity graphs at the LV base (panel A), mid-ventricle (panel B) and LV apex (panel C).

Positive values reflect clockwise rotation and negative values reflect counter-clockwise rotation as viewed from the apex. Solid line - patients with CAD; Dotted line - healthy controls. In healthy volunteers, the circumferential velocity graph at the LV apex showed an undulating pattern of alternating waves of counter-clockwise rotation (panel C, dotted line, waves a, $c$ and $e$ ) and waves of sudden deceleration or opposite rotations (waves b, $d$ and f). In CAD patients, these waves of alternating rotational motion were less prominent (panel $C$, solid line, waves a-h). An altered pattern of ventricular untwisting was also observed in patients with $C A D$, with much higher peak clockwise velocities at the LV apex (wave h). Cardiac cycle phases: 1 - isovolumetric contraction; 2 - rapid ejection; 3 - reduced ejection; 4 - isovolumetric relaxation; 5 - rapid filling; 6 - diastasis; and 7 - atrial systole; ED: End Diastole; ES: End Systole

The circumferential velocities for individual ventricular segments at the LV base, mid-ventricle and LV apex are displayed in figure 6,7 and 8 respectively. An altered pattern of rotational motion is observed in patients with CAD, particularly affecting apical and, to a lower degree, mid-ventricular and basal LV segments.

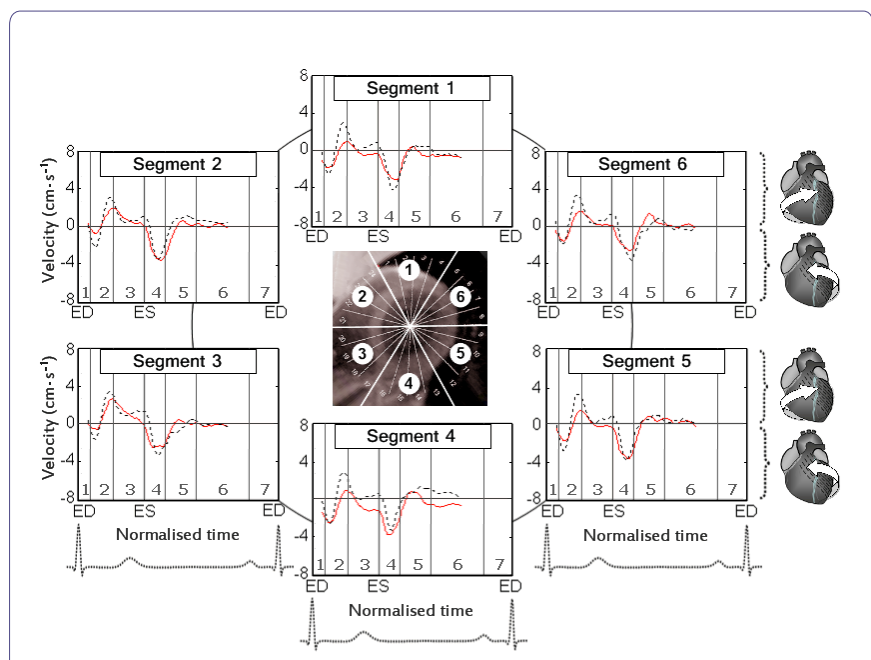

Figure 6: Circumferential velocity graphs for basal LV segments during a cardiac cycle.

Solid line - patients with CAD; Dotted line - healthy controls; Segments: 1 - basal anterior segment; 2 - basal anteroseptal segment; 3 - basal inferoseptal segment; 4 - basal inferior segment; 5 - basal inferolateral segment; and 6 - basal anterolateral segment; Cardiac cycle phases: 1 - isovolumetric contraction; 2 - rapid ejection; 3 - reduced ejection; 4 - isovolumetric relaxation; 5 - rapid filling; 6 - diastasis; and 7 - atrial systole; ED: End Diastole; ES: End Systole 


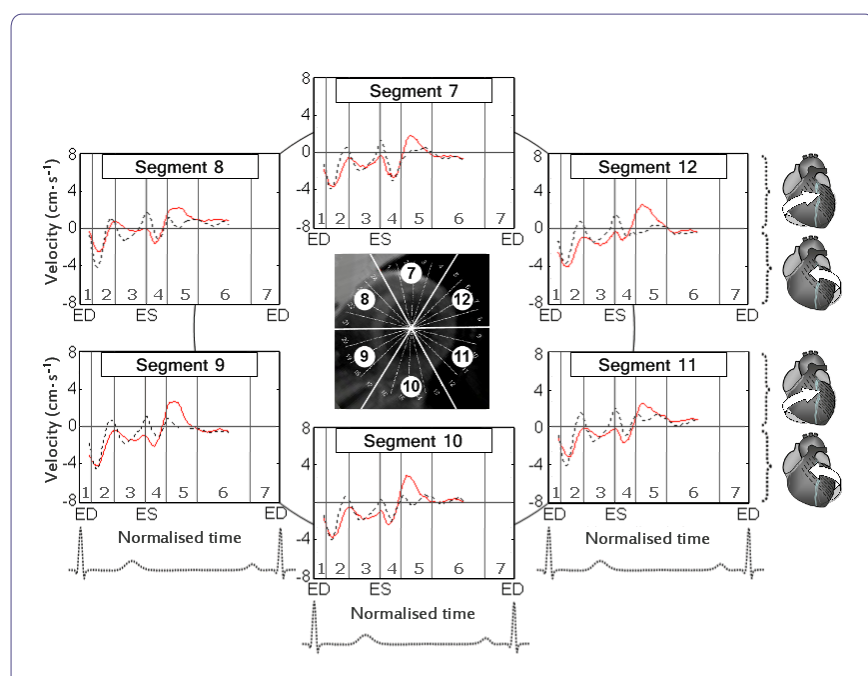

Figure 7: Circumferential velocity graphs for mid-ventricular segments during a cardiac cycle.

Solid line - patients with CAD; Dotted line - healthy controls; Segments: 7 - mid-anterior segment; 8 - mid-anteroseptal segment; 9 - mid-inferoseptal segment; 10 - mid-inferior segment; 11 - mid-inferolateral segment; 12 - mid-anterolateral segment; Cardiac cycle phases: 1 - isovolumetric contraction; 2 - rapid ejection; 3 - reduced ejection; 4 - isovolumetric relaxation; 5 - rapid filling; 6 - diastasis; and 7 - atrial systole; ED: End Diastole; ES: End Systole

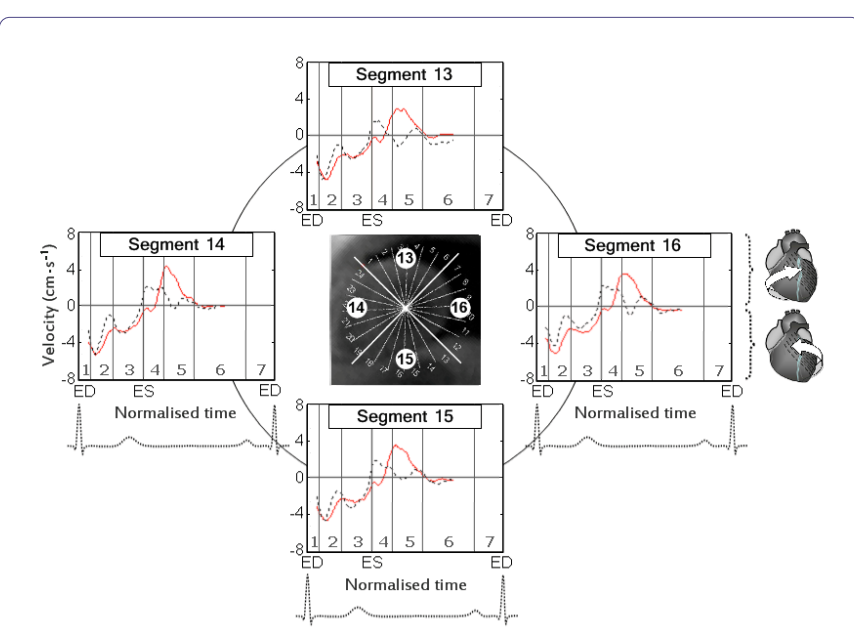

Figure 8: Circumferential velocity graphs for apical LV segments during a cardiac cycle.

Solid line - patients with CAD; Dotted line - healthy controls. A changed pattern of rotational motion of all apical segments was distinctly noted in CAD group. Segments: 13 - apical anterior segment; 14 - apical septal segment; 15 - apical inferior segment; and 16 - apical lateral segment; Cardiac cycle phases: 1 - isovolumetric contraction; 2 - rapid ejection; 3 - reduced ejection; 4 - isovolumetric relaxation; 5 - rapid filling; 6 - diastasis; 7 - atrial systole; ED: End Diastole; ES: End Systole

\section{Longitudinal motion}

Peak longitudinal velocities with corresponding time to peak values for main LV slices are presented in table 3 , while the graphical display is provided in figure 9 (a - peak systolic longitudinal velocity, b - peak diastolic longitudinal velocity). Positive values demonstrate downward motion along the longitudinal LV axis (i.e., towards the apex) and negative values show upward motion (i.e., towards the base). Peak systolic longitudinal velocities in both
CAD patients and healthy controls were represented by the downward motion of the left ventricle during rapid ejection (Figure 9, arrow a), when the entire ventricle was pushed in an opposite direction to the ejected blood. The values were much lower in CAD patients, being statistically significant at all levels (Table 3 ). Peak diastolic velocities in both groups were represented by a recoil wave of upward motion in diastole (Figure 9, arrow b). The peak diastolic longitudinal velocities at the LV base in CAD patients were almost half of those recorded in healthy controls (Table 3), but the difference decreased towards the apex. No difference was noted in the time to peak velocity values (Table 3).

The longitudinal velocities for individual ventricular segments at the LV base, mid-ventricle and LV apex are displayed in figure 10,11 and 12 respectively. Since the apical segments (i.e., segments 13-16) were relatively stable with little motion along LV longitudinal axis even in healthy controls, the biggest difference between the two groups was recorded in longitudinal motion of basal LV segments (i.e., segments $1-6)$.

\section{Left ventricular torsion rate and longitudinal strain rate}

The peak systolic torsion rate was significantly lower in patients with CAD compared with healthy controls $(15.1 \pm 1.0$ versus $20.6 \pm 2.0$ degrees/s/cm, $p$-value 0.01 , Table 4 and Figure 13). A lower peak diastolic torsion rate was also apparent in CAD group $(-19.6 \pm 2.1$ versus $-25.2 \pm 1.8$ degrees $/ \mathrm{s} / \mathrm{cm})$, although the difference did not reach statistical significance in our study $(p$-value $=0.12)$. The greatest difference between CAD and control subjects, however, was recorded for peak diastolic longitudinal strain rate values, which were approximately 2.5 times lower in patients with CAD $(1.1 \pm 0.2$ versus $2.7 \pm 0.1 \mathrm{~s}^{-1}, p$-value $\left.<0.01\right)$ (Table 4 and Figure 14). The difference between the two groups was also significant for peak systolic longitudinal strain rate $(p$-value $<0.01)$.

\section{Discussion}

This study revealed prominent differences in radial, rotational and longitudinal motion patterns of all myocardial layers, suggesting significant LV dysfunction associated to CAD.

\section{Reduced radial ventricular wall motion in CAD}

The lower systolic radial velocities in the CAD group (Table 1) underscored decreased inward LV contractility in these patients, with contraction of LV apical segments being affected to the greatest extent. The findings are consistent with previous reports showing a greater dysfunction in distal compared to proximal myocardial segments following induced ischemia in both animal and human studies $[25,26]$. Radial expansion in diastole was affected even more than inward motion in systole, involving also the LV base in patients with CAD (Table 1). Meanwhile, there was little change in the time to peak radial velocities, indicating these times are not informative for the assessment of CAD. The greatest difference between peak radial velocities was recorded for the inner/endocardial layer of the myocardium, which is consistent with reports that subendocardial layer receives more blood flow and has a higher energy requirement $[11,27]$, being therefore more sensitive to ischemic changes [11,28,29]. In support of this hypothesis, graded ischemia applied in experimental studies also affected initially the subendocardium before extending to the midwall and epicardial layers $[1,30]$. In patients with severe CAD, the reduced velocities at the subendocardial level can be also related to subendocardial fibrosis and perhaps subendocardial necrosis caused 
Citation: Codreanu I, Pegg TJ, Selvanayagam JB, Robson MD, Rider OJ, et al. (2015) Comprehensive Assessment of Left Ventricular Wall Motion Abnormalities in Coronary Artery Disease Using Cardiac Magnetic Resonance. J Cardiol Stud Res 2: 006.

- Page 7 of $11 \bullet$

\begin{tabular}{|c|c|c|c|c|c|c|c|}
\hline \multicolumn{2}{|c|}{$\begin{array}{c}\text { LV level and myocardial } \\
\text { layer }\end{array}$} & CAD patients & Healthy controls & $p$-value & CAD patients & Healthy controls & $p$-value \\
\hline \multicolumn{5}{|c|}{ Peak systolic longitudinal velocity $(\mathrm{cm} / \mathrm{s})$} & \multicolumn{3}{|c|}{ Time to peak values (\% of ES) } \\
\hline \multirow{3}{*}{ LV base } & epicardial & $5.8 \pm 0.5$ & $7.8 \pm 1.0$ & 0.02 & $29.5 \pm 1.6$ & $26.0 \pm 1.2$ & 0.14 \\
\hline & transmural & $5.5 \pm 0.5$ & $7.9 \pm 0.9$ & 0.01 & $29.8 \pm 1.6$ & $25.5 \pm 1.1$ & 0.07 \\
\hline & endocardial & $5.3 \pm 0.5$ & $8.0 \pm 0.9$ & $<0.01$ & $29.5 \pm 1.8$ & $25.0 \pm 0.9$ & 0.08 \\
\hline \multirow{3}{*}{ Mid-ventricle } & epicardial & $4.5 \pm 0.4$ & $6.0 \pm 0.6$ & 0.03 & $28.1 \pm 1.4$ & $25.1 \pm 1.2$ & 0.20 \\
\hline & transmural & $4.0 \pm 0.4$ & $5.9 \pm 0.5$ & $<0.01$ & $28.1 \pm 1.4$ & $25.7 \pm 1.3$ & 0.31 \\
\hline & endocardial & $3.6 \pm 0.5$ & $5.8 \pm 0.5$ & $<0.01$ & $28.4 \pm 1.3$ & $25.7 \pm 1.3$ & 0.24 \\
\hline \multirow{3}{*}{ LV apex } & epicardial & $2.2 \pm 0.4$ & $3.9 \pm 0.6$ & 0.01 & $26.6 \pm 1.8$ & $25.8 \pm 1.5$ & 0.77 \\
\hline & transmural & $2.0 \pm 0.4$ & $3.7 \pm 0.6$ & 0.02 & $26.6 \pm 1.8$ & $25.8 \pm 1.5$ & 0.77 \\
\hline & endocardial & $1.9 \pm 0.5$ & $3.5 \pm 0.7$ & 0.04 & $26.9 \pm 1.7$ & $25.8 \pm 1.5$ & 0.68 \\
\hline \multicolumn{5}{|c|}{ Peak diastolic longitudinal velocity $(\mathrm{cm} / \mathrm{s})$} & \multicolumn{3}{|c|}{ Time to peak values (\% of ED) } \\
\hline \multirow[t]{2}{*}{ LV base } & transmural & $-7.9 \pm 0.6$ & $-14.1 \pm 1.1$ & $<0.01$ & $22.9 \pm 1.2$ & $20.6 \pm 0.8$ & 0.26 \\
\hline & endocardial & $-8.0 \pm 0.6$ & $-14.7 \pm 1.2$ & $<0.01$ & $23.5 \pm 1.3$ & $20.1 \pm 0.7$ & 0.14 \\
\hline \multirow{3}{*}{ Mid-ventricle } & epicardial & $-7.0 \pm 0.5$ & $-10.6 \pm 0.7$ & $<0.01$ & $23.5 \pm 1.2$ & $24.4 \pm 0.8$ & 0.69 \\
\hline & transmural & $-6.6 \pm 0.5$ & $-10.7 \pm 0.7$ & $<0.01$ & $23.7 \pm 1.4$ & $24.4 \pm 0.8$ & 0.78 \\
\hline & endocardial & $-6.6 \pm 0.6$ & $-10.7 \pm 0.7$ & $<0.01$ & $24.0 \pm 1.6$ & $24.1 \pm 0.8$ & 0.99 \\
\hline \multirow{3}{*}{ LV apex } & epicardial & $-6.1 \pm 0.5$ & $-7.0 \pm 0.6$ & 0.20 & $21.1 \pm 1.4$ & $23.0 \pm 2.0$ & 0.52 \\
\hline & transmural & $-6.0 \pm 0.6$ & $-6.9 \pm 0.8$ & 0.18 & $20.2 \pm 1.4$ & $23.0 \pm 2.0$ & 0.33 \\
\hline & endocardial & $-6.1 \pm 0.6$ & $-6.8 \pm 0.7$ & 0.23 & $20.3 \pm 1.4$ & $20.7 \pm 2.2$ & 0.91 \\
\hline
\end{tabular}

Table 3: Peak longitudinal velocities and time to peak values in patients with $C A D$ and healthy controls*.

${ }^{*}$ All values are presented as mean \pm SEM

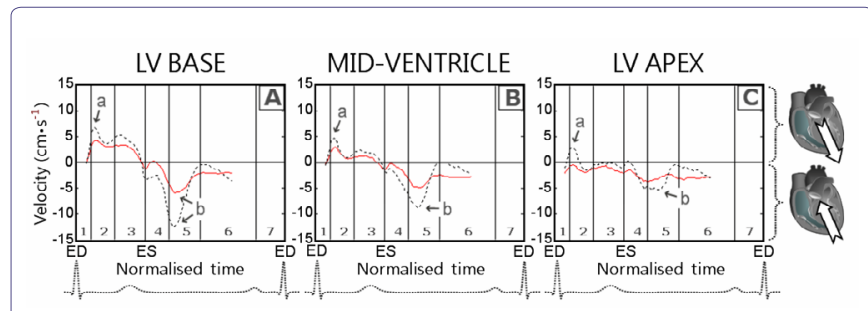

Figure 9: Longitudinal velocity graphs at the LV base (panel A), mid-ventricle (panel B) and LV apex (panel C).

Positive values demonstrate downward motion along the longitudinal LV axis (i.e., towards the apex) and negative values show upward motion (i.e., towards the base). Solid line - patients with CAD; dotted line - healthy controls. Arrows point towards peak systolic (a) and peak diastolic (b) longitudinal velocities. Cardiac cycle phases: 1 - isovolumetric contraction; 2 - rapid ejection; 3 - reduced ejection; 4 - isovolumetric relaxation; 5 - rapid filling; 6 - diastasis; and 7 - atrial systole; ED: End Diastole; ES: End Systole

by severe, chronic subendocardial ischemia [31]. It is well known that such lesions are commonly localized in the subendocardium, while non-ischemic cardiomyopathies often display an intramyocardial or subepicardial pattern [32].

\section{Alterations in left ventricular rotation in CAD}

The circumferential velocity traces demonstrated not only differences in peak velocities, but also an altered pattern of rotational motion. The higher initial counterclockwise velocities obtained in CAD are consistent with experiments of Kroeker et al., [33], who showed that ischemia induced by a short period of coronary artery occlusion in animal models resulted in increased counter-clockwise rotation. As a potential explanation the authors suggested that subendocardial ischemia led to a loss of counteraction

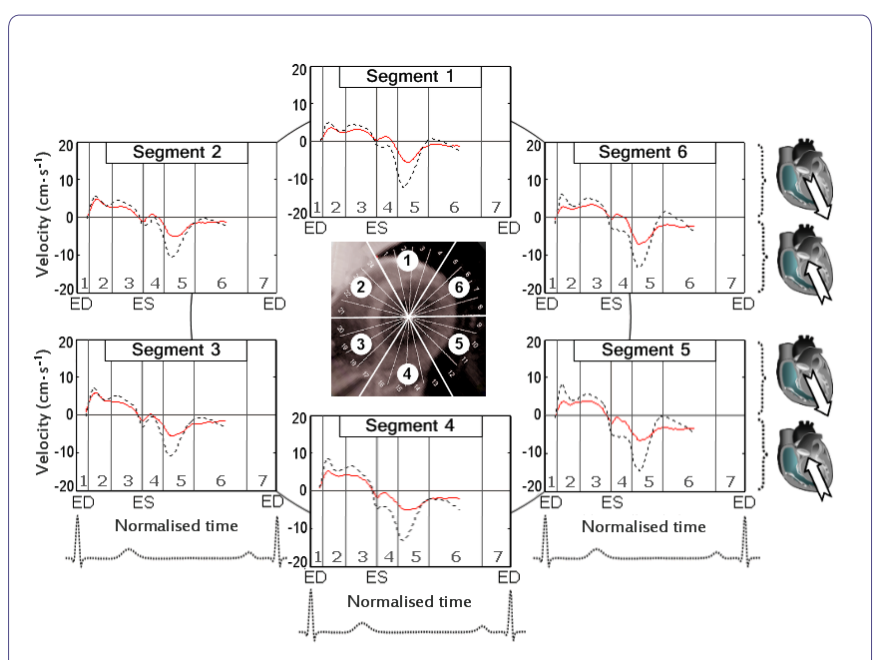

Figure 10: Longitudinal velocity graphs for basal LV segments during a cardiac cycle.

Solid line - patients with CAD; Dotted line - healthy controls; Segments: 1 - basal anterior segment; 2 - basal anteroseptal segment; 3 - basal inferoseptal segment; 4 - basal inferior segment; 5 - basal inferolateral segment; 6 - basal anterolateral segment; Cardiac cycle phases: 1 - isovolumetric contraction; 2 - rapid ejection; 3 - reduced ejection; 4 - isovolumetric relaxation; 5 - rapid filling; 6 - diastasis; and 7 - atrial systole; ED: End Diastole; ES: End Systole

of contraction of subendocardial cardiomyocytes, which have a clockwise orientation $[33,34]$.

The delay in LV apical untwisting in CAD subjects, made the clockwise rotation of the LV apex (Figure $5 \mathrm{C}$, wave $\mathrm{h}$, solid line) coincide at least partially with the clockwise rotation of the LV base 


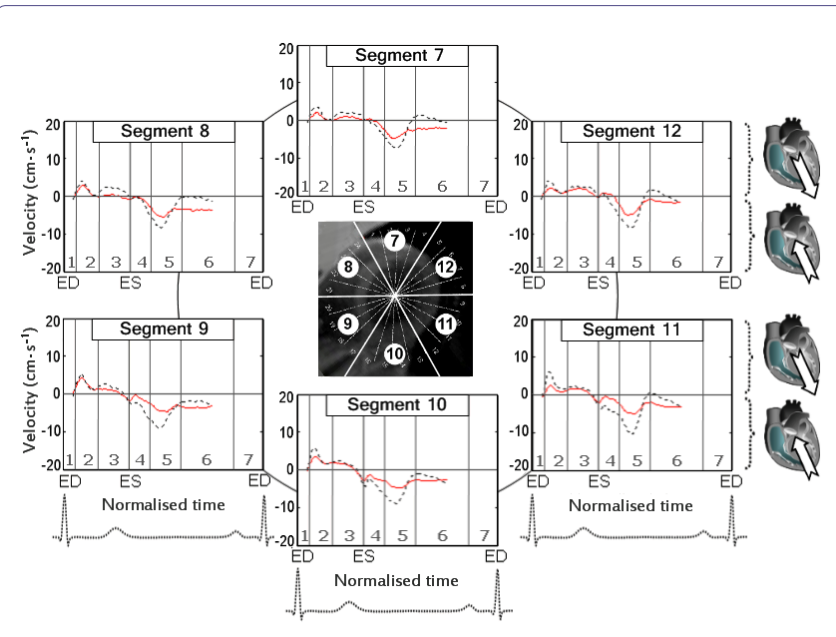

Figure 11: Longitudinal velocity graphs for mid-ventricular segments during a cardiac cycle.

Solid line - patients with CAD; Dotted line - healthy controls; Segments: 7 - mid-anterior segment; 8 - mid-anteroseptal segment: 9 - mid-inferoseptal segment; 10 - mid-inferior segment; 11 - mid-inferolateral segment; 12 - mid-anterolateral segment; Cardiac cycle phases: 1 - isovolumetric contraction; 2 - rapid ejection; 3 - reduced ejection; 4 - isovolumetric relaxation; 5 - rapid filling; 6 - diastasis; 7 - atrial systole; ED: End Diastole; ES: End Systole

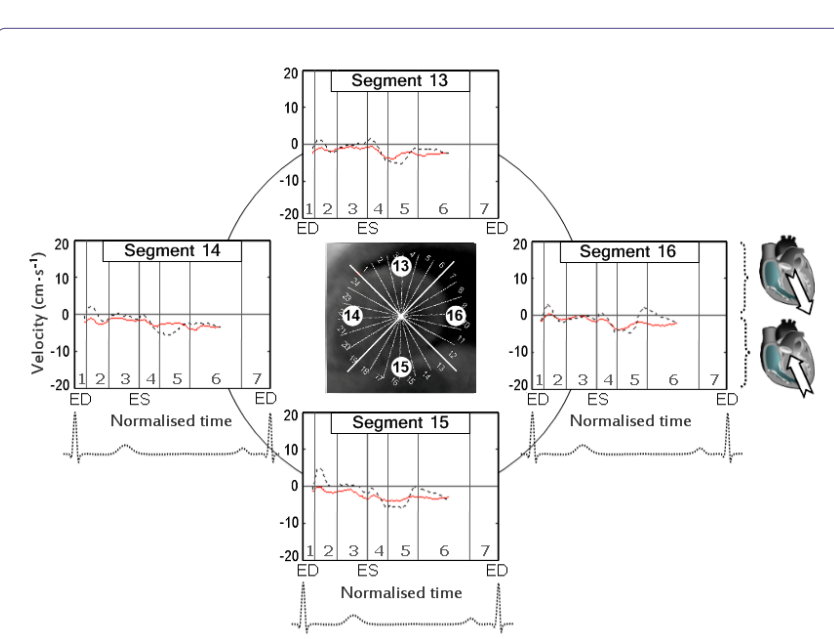

Figure 12: Longitudinal velocity graphs for apical LV segments during a cardiac cycle.

Solid line - patients with CAD; Dotted line - healthy controls. Since apical segments showed little motion along longitudinal LV axis even in healthy controls, the biggest difference between the two groups was noted in longitudinal motion of the basal LV segments (Figure 10). Segments: 13 - apical anterior segment; 14 - apical septal segment; 15 - apical inferior segment; 16 - apical lateral segment; Cardiac cycle phases: 1 - isovolumetric contraction; 2 - rapid ejection; 3 - reduced ejection; 4 - isovolumetric relaxation; 5 - rapid filling; 6 - diastasis; 7 - atrial systole; ED: End Diastole; ES: End Systole

(Figure 5A, wave $\mathrm{f}$, solid line). This concordance in motion direction of the LV apex with the massive LV base may explain the much higher peak clockwise velocities at the LV apex (and even mid-ventricle) recorded in patients with CAD (Table 2 and Figure $5 \mathrm{C}$, wave $\mathrm{h}$, solid line). The altered rotational pattern of the $\mathrm{LV}$ apex might also explain the higher peak counter-clockwise velocities at the LV base (despite lower peak clockwise values) recorded in CAD patients. During the first half of isovolumetric relaxation, the LV apex in healthy subjects was rotating in a clockwise direction (Figure 5C,

\begin{tabular}{|l|c|c|c|}
\hline & CAD & Controls & $\boldsymbol{p}$-value \\
\hline Peak systolic torsion rate (degrees/s/cm) & $15.1 \pm 1.0$ & $20.6 \pm 2.0$ & 0.01 \\
\hline $\begin{array}{l}\text { Time to peak systolic torsion rate }(\% \text { of end } \\
\text { systole) }\end{array}$ & $43.2 \pm 2.2$ & $45.9 \pm 3.9$ & 0.56 \\
\hline Peak diastolic torsion rate (degrees/s/cm) & $-19.6 \pm 2.1$ & $-25.2 \pm 1.8$ & 0.12 \\
\hline $\begin{array}{l}\text { Time to peak diastolic torsion rate (per cent } \\
\text { of end systole) }\end{array}$ & $133.8 \pm 2.7$ & $122.7 \pm 2.0$ & 0.01 \\
\hline Peak systolic longitudinal strain rate $\left(\mathrm{s}^{-1}\right)$ & $-1.3 \pm 0.1$ & $-1.8 \pm 0.2$ & $<0.01$ \\
\hline $\begin{array}{l}\text { Time to peak systolic longitudinal strain rate } \\
\text { (\% of end systole) }\end{array}$ & $44.6 \pm 3.5$ & $48.0 \pm 3.2$ & 0.60 \\
\hline Peak diastolic longitudinal strain rate $\left(\mathrm{s}^{-1}\right)$ & $1.1 \pm 0.2$ & $2.7 \pm 0.1$ & $<0.01$ \\
\hline $\begin{array}{l}\text { Time to peak diastolic longitudinal strain rate } \\
\text { (\% of end systole) }\end{array}$ & $147.8 \pm 2.5$ & $134.4 \pm 4.2$ & 0.02 \\
\hline
\end{tabular}

Table 4: Peak ventricular torsion rate and peak longitudinal strain rate in patients with CAD and healthy controls*.

${ }^{*}$ All values are presented as mean \pm SEM

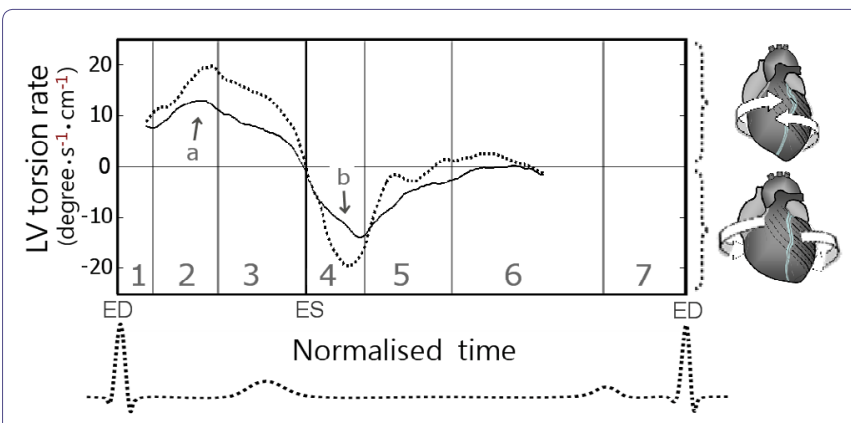

Figure 13: Global LV torsion rate during a cardiac cycle.

Systolic torsion, expressed in positive values, results from a clockwise rotation of LV base and a simultaneous counterclockwise rotation of the LV apex, while torsion rate represents the speed at which the twisting motion occurs. Subsequent recoil of twist deformation following ventricular repolarization is associated with a clockwise rotation of LV apex and a simultaneous counterclockwise rotation of the LV base (reversed motions), translating into negative values of torsion rate. The arrows show peak systolic (a) and peak diastolic (b) torsion rates. Solid line - CAD patients; Dotted line - healthy controls; Cardiac cycle phases: 1 - isovolumetric contraction; 2 - rapid ejection; 3 - reduced ejection; 4 - isovolumetric relaxation; 5 - rapid filling; 6 - diastasis; 7 - atrial systole; ED: End Diastole; ES: End Systole

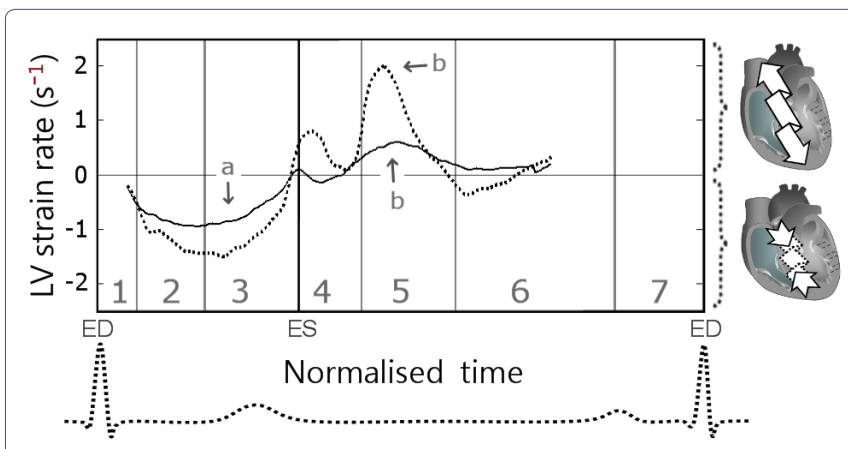

Figure 14: Longitudinal LV strain rate during a cardiac cycle.

Positive values correspond to LV lengthening, where negative values indicate LV shortening. The arrows show peak systolic (a) and peak diastolic (b) longitudinal strain rates. Solid line - CAD patients; Dotted line - healthy controls; Cardiac cycle phases: 1 - isovolumetric contraction; 2 - rapid ejection; 3 - reduced ejection; 4 - isovolumetric relaxation; 5 - rapid filling; 6 - diastasis; 7 - atrial systole; ED: End Diastole; ES: End Systole

wave $\mathrm{d}$, dotted line), which was opposite to the rotation of the LV base (Figure 5A, wave e, dotted line). In CAD patients, however, the LV apex was still rotating counter-clockwise during the first half of 
isovolumetric relaxation, the velocity trace showing negative values (Figure 5C, solid line). Thus, in healthy controls, the LV base and apex were rotating in opposite directions, whilst in CAD patients the counter-clockwise rotational recoil of the LV base encountered little or no resistance from the LV apex, resulting in higher peak values. These findings demonstrate how an altered rotational pattern of the ventricular apex can directly affect the rotational motion of the LV base. Significant alterations of LV rotational pattern were also noted during the phase of rapid ventricular filling, when the LV apex was rotating clockwise in CAD patients (Figure $5 \mathrm{C}$, wave $\mathrm{h}$ ) and counter-clockwise in healthy controls (Figure 5C, wave e). Most of these alterations of rotational pattern are expected to directly affect the ventricular physiology and LV filling. Correlative studies involving ventricular hemodynamics may shed new light into these mechanisms.

To provide a better understanding of the altered pattern of rotational motion of the LV apex and base against each other, we compared the circumferential velocity graphs with those obtained in a younger age group (healthy controls $23 \pm 3$ years old from an unrelated study [17]) (Figure 15). The graphs showed successive changes, with initial alterations of the rotational motions of the left ventricle in older age group and subsequent progressive loss of the undulating pattern of ventricular twisting in patients with CAD. Of particular note is that the peak counter-clockwise velocity at the $\mathrm{LV}$ base in younger subjects was represented by the initial wave of counter-clockwise rotation of the entire ventricle at the commencement of systole (Figure 15A, solid line, wave b), whilst in the older age group, as well as in patients with CAD it was represented by a recoil wave of ventricular untwisting in diastole (Figure 15B and C, solid line, wave b).

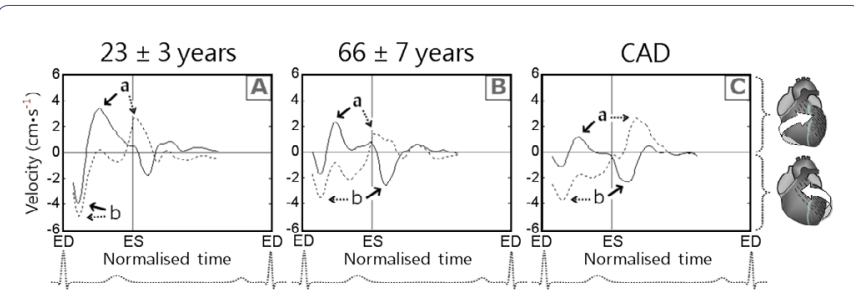

Figure 15: Changes in LV rotational motion pattern with increasing age $(A, B)$ and in patients with $C A D(C)$.

Solid line - rotational motion of the LV base; Dashed line - rotational motion of the LV apex. Positive values reflect clockwise rotation and negative values reflect counter-clockwise rotation as viewed from the apex. The arrows point towards the peak clockwise (a) and peak counter-clockwise (b) velocities. Successive changes, with a progressive loss of the undulating pattern of rotational motions can be noted. Of note is that the peak counter-clockwise velocity at the LV base (b, solid graph) in younger subjects was represented by the initial wave of counter-clockwise rotation of the entire ventricle at the commencement of systole, whilst in the older age group and patients with CAD it was represented by a recoil wave of ventricular untwisting in diastole. ED: End Diastole; ES: End Systole

\section{Pronounced alterations in ventricular longitudinal motion in CAD}

The greatest difference between myocardial velocities of CAD patients and healthy controls was found in ventricular longitudinal velocities (Figure 9). Hence, determination of longitudinal velocities may be one of the most sensitive tests for evaluation of myocardial contractility. During systole, the entire ventricle was pushed in an opposite direction to the ejected blood, synonymous to the recoil of a canon after being fired. This motion pattern was seen as a wave of downward displacement at all LV levels (arrow a, Figure 9). The amplitude of this longitudinal motion is expected to be proportional to the force of the ejected blood and, consequently, to the summation of all elements of LV contraction, including radial and rotational motions. For example, altered myocardial contractility is likely to result in a lower radial velocity and contractility; altered rotational motion is expected to affect the force of LV twisting; potential myocardial scars might result in akinetic and dyskinetic regions. All these factors potentially affect the overall force of the ejected blood and, consequently, the force with which the ventricle is being pushed in an opposite direction, stretching the great vessels and elastic structures to which it is attached. After repolarization, these stretched elastic structures start pulling the displaced ventricle (i.e., "fired canon") upward towards its initial location. The velocity of this longitudinal recoil motion (arrow b, Figure 9) was expected to be proportional to the overall force applied during systole to stretch the great vessels and elastic structures attaching the ventricle to mediastinum. Consequently, peak diastolic longitudinal velocity can serve as a measure of global ventricular contractility, the resulted recoil in diastole being proportional to the sum of all applied forces during systole. In addition, peak diastolic longitudinal velocity represents the highest velocity of the myocardial wall. The highest longitudinal velocities recorded in this study exceeded $-14 \mathrm{~cm} / \mathrm{s}$ (Table 3), while the highest radial and circumferential velocities were $-6.6 \mathrm{~cm} / \mathrm{s}$ (Table 1) and $-4.7 \mathrm{~cm} / \mathrm{s}$ (Table 2), respectively. In a failing heart, one might expect to see the greatest difference in the fastest myocardial motions, i.e., in diastolic longitudinal velocities. The findings are consistent with previous reports that the amplitude of long axis motion provides a useful approximation of the ejection fraction [35].

Since the ventricular apex shows little motion along the longitudinal axis, due to concomitant LV shortening/twisting in systole and elongation/untwisting in diastole, the greatest difference in longitudinal motion can be expected at the LV base. In this study, the peak longitudinal velocities at the LV base were approximately two times lower in CAD patients compared with the values registered in healthy controls, representing the most significant difference between the two groups (Figure 9A, wave b). These findings were confirmed by similar differences in longitudinal strain rates (Table 4 and Figure 14). Our results are concordant with the findings reported in patients with acute myocardial ischemia, indicating reduced global longitudinal strain as the single most powerful marker of manifest left ventricular hemodynamic deterioration in the acute phase of myocardial infarction [36]. After the acute phase, longitudinal strain also proved an important predictor for segmental and global LV function improvement [37]. Furthermore, peak systolic longitudinal strain at rest was reported useful in predicting the presence of left main and three vessel coronary artery disease even before the development of regional wall motion abnormalities [38].

We also believe the findings would complement our previous studies [16,17], providing a broader understanding of underlying changes in left ventricular wall motion in elderly individuals, patients with coronary artery disease and subsequent ventricular remodeling following revascularization.

\section{Study limitations and perspectives}

The cardiac phases were calculated and normalized for the entire group based on their average duration during systole and diastole and not on the timing of cardiac valves closure and opening, which was not recorded. The phases are provided for general orientation and even when slightly displaced would not affect data interpretation. 
Visual assessment of individual segmental radial, circumferential and longitudinal velocity graphs revealed no significant difference to account for a particular motion pattern in any subject (e.g., large scars, regional paradoxical motion, etc.). Since all patients included in the study had triple-vessel coronary artery disease, no significant difference averaged between affected myocardial regions was expected. Given the number of resulting parameters (16 segments x 3 myocardial layers x ( 3 velocities +3 time to peak values $)=288$ parameters), no myocardial velocities for individual segments were calculated in this case, the evaluation being more suitable for patients with regional rather than global CAD. Mechanical tethering of the adjacent segments to infarcted or ischemic regions has also been described [39-41]. In a cohort of patients with triple vessel disease and scattered affected areas throughout the entire LV, evaluating regional motion of the effected LV segments becomes especially challenging. Evaluating segmental velocities and regional myocardial motion in patients with a single-vessel coronary disease and correlating the affected coronary artery with recorded regional wall motion abnormalities may represent an area of interest for further research. Calculating the regional strain and strain rates of the affected LV segments would provide additional insight into regional LV wall motion in such patients $[42,43]$. Furthermore, correlating the TPM analysis technique with late gadolinium enhancement studies may provide new details about the impact of myocardial fibrosis and infarcted areas on regional and global LV mechanics. This study was also limited to the assessment of ventricular wall motion only. It has been reported that LV wall motion analysis combined with ECG changes and serum markers of myocardial necrosis may allow recognition of stress cardiomyopathies in different clinical settings [44]. In the current study, the endocardial and myocardial borders were delineated manually, being rather time consuming. Programs for automatic delineation that can facilitate the clinical applications of the technique are currently under development. Correlation with other imaging modalities, including with speckle tracking echocardiography in larger populations may further define the suitability and clinical role of TPM among the existing cardiac investigations.

\section{Conclusion}

The study revealed significant differences in radial, rotational and longitudinal LV motions as well as changes within myocardial layers in patients with CAD. The changes were more pronounced towards the LV apex, the sub-endocardial myocardium being affected to a greater extent. An altered pattern of ventricular rotational motion was also observed. The greatest difference between the two groups was noted in the longitudinal recoil motion of the left ventricle during diastole. Consequently, peak diastolic longitudinal velocity at the LV base and peak diastolic longitudinal strain rate might represent the most sensitive parameters in assessing global LV contractility. Further studies are needed in single and multivessel disease to determine whether these parameters provide important diagnostic and prognostic information for patients suffering from CAD.

\section{Acknowledgements}

This research was supported by British Heart Foundation grant number RG/07/004/22659.

\section{References}

1. Moen CA, Salminen PR, Grong K, Matre K (2011) Left ventricular strain, rotation, and torsion as markers of acute myocardial ischemia. Am J Physiol Heart Circ Physiol 300: 2142-2154.

2. Mahenthiran J, Das MK, Bhakta D, Ghumman W, Feigenbaum H, et al (2006) Prognostic importance of wall motion abnormalities in patients with ischemic cardiomyopathy and an implantable cardioverter-defibrillator. Am J Cardiol 98: 1301-1306.

3. Sanderson JE, Wang M, Yu CM (2004) Tissue Doppler imaging for predicting outcome in patients with cardiovascular disease. Curr Opin Cardiol 19: 458-463.

4. Safford RE, Bove AA (1987) Prediction of coronary artery disease by left ventricular regional wall motion abnormalities in patients with stenosis of the aortic valve. Br Heart J 57: 237-241.

5. Yuda S, Fang ZY, Marwick TH (2003) Association of severe coronary stenosis with subclinical left ventricular dysfunction in the absence of infarction. J Am Soc Echocardiogr 16: 1163-1170.

6. Launbjerg J, Berning J, Fruergaard P, Eliasen P, Borch-Johnsen K, et al. (1992) Risk stratification after acute myocardial infarction by means of echocardiographic wall motion scoring and Killip classification. Cardiology 80: 375-381.

7. Carluccio E, Tommasi S, Bentivoglio M, Buccolieri M, Prosciutti L, et al. (2000) Usefulness of the severity and extent of wall motion abnormalities as prognostic markers of an adverse outcome after a first myocardial infarction treated with thrombolytic therapy. Am J Cardiol 85: 411-415.

8. Kjøller E, Kober L, Jørgensen S, Torp-Pedersen C (2000) Short and long term prognostic importance of regional dyskinesia versus akinesia in acute myocardial infarction. Heart 87: 410-414.

9. Herz SL, Hasegawa T, Makaryus AN, Parker KM, Homma S, et al. (2010) Quantitative three-dimensional wall motion analysis predicts ischemic region size and location. Ann Biomed Eng 38: 1367-1376.

10. Li XC, Jin FL, Jing C, Xiao Q, Liu Y, et al. (2012) Predictive value of left ventricular remodeling by area strain based on three-dimensional wall-motion tracking after $\mathrm{PCl}$ in patients with recent NSTEMI. Ultrasound Med Biol 38: 1491-1501.

11. Ishizu T, Seo Y, Baba M, Machino T, Higuchi H, et al. (2011) Impaired subendocardial wall thickening and post-systolic shortening are signs of critical myocardial ischemia in patients with flow-limiting coronary stenosis. Circ J 75: 1934-1941.

12. Hauser AM, Gangadharan V, Ramos RG, Gordon S, Timmis GC (1985) Sequence of mechanical, electrocardiographic and clinical effects of repeated coronary artery occlusion in human beings: echocardiographic observations during coronary angioplasty. J Am Coll Cardiol 5: 193-197.

13. Hoffmann R, Altiok E, Nowak B, Heussen N, Kühl H, et al. (2002) Strain rate measurement by doppler echocardiography allows improved assessment of myocardial viability inpatients with depressed left ventricular function. J Am Coll Cardiol 39: 443-449.

14. Grabka M, Wita K, Tabor Z, Paraniak-Gieszczyk B, Chmurawa J, et al. (2013) Prediction of infarct size by speckle tracking echocardiography in patients with anterior myocardial infarction. Coron Artery Dis 24: 127-134.

15. Yang J, Liu X, Jiang G, Chen Y, Zhang Y, et al. (2012) Two-dimensional strain technique to detect the function of coronary collateral circulation. Coron Artery Dis 23: 188-194.

16. Codreanu I, Pegg TJ, Selvanayagam JB, Robson MD, Rider OJ, et al. (2011) Details of left ventricular remodeling and the mechanism of paradoxical ventricular septal motion after coronary artery bypass graft surgery. J Invasive Cardiol 23: 276-282.

17. Codreanu I, Pegg TJ, Selvanayagam JB, Robson MD, Rider OJ, et al. (2014) Normal values of regional and global myocardial wall motion in young and elderly individuals using navigator gated tissue phase mapping. Age (Dordr) 36: 231-241. 
18. Jung B, Föll D, Böttler P, Petersen S, Hennig J, et al. (2006) Detailed analysis of myocardial motion in volunteers and patients using high-temporal-resolution MR tissue phase mapping. J Magn Reson Imaging 24: 1033-1039.

19. Jung B, Markl M, Föll D, Hennig J (2006) Investigating myocardial motion by MRI using tissue phase mapping. Eur J Cardiothorac Surg 29: 150-157.

20. Codreanu I, Robson MD, Golding SJ, Jung BA, Clarke K, et al. (2010) Longitudinally and circumferentially directed movements of the left ventricle studied by cardiovascular magnetic resonance phase contrast velocity mapping. J Cardiovasc Magn Reson 12: 48.

21. Codreanu I, Robson MD, Rider OJ, Pegg TJ, Jung BA, et al. (2011) Chasing the reflected wave back into the heart: a new hypothesis while the jury is still out. Vasc Health Risk Manag 7: 365-373.

22. Codreanu I, Robson MD, Rider OJ, Pegg TJ, Dasanu CA, et al. (2013) Effects of ventricular insertion sites on rotational motion of left ventricular segments studied by cardiac MR. Br J Radiol 86: 20130326.

23. Codreanu I, Robson MD, Rider OJ, Pegg TJ, Dasanu CA, et al. (2014) Details of left ventricular radial wall motion supporting the ventricular theory of the third heart sound obtained by cardiac MR. Br J Radiol 17: 20130780.

24. Cerqueira MD, Weissman NJ, Dilsizian V, Jacobs AK, Kaul S, et al. (2002) Standardized myocardial segmentation and nomenclature for tomographic imaging of the heart: a statement for healthcare professionals from the Cardiac Imaging Committee of the Council on Clinical Cardiology of the American Heart Association. Circulation 105: 539-542.

25. Ishii K, Suyama T, Imai M, Maenaka M, Yamanaka A, et al. (2009) Abnormal regional left ventricular systolic and diastolic function in patients with coronary artery disease undergoing percutaneous coronary intervention: clinical significance of post-ischemic diastolic stunning. J Am Coll Cardiol 54: 1589-1597.

26. Hu N, Sabey KH, Curtis HR, Hoang N, Dowdle SB, et al. (2012) Magnetic Resonance Imaging (MRI) assessment of ventricular remodeling after myocardial infarction in rabbits. Comp Med 62: 116-123.

27. Oh BH, Volpini M, Kambayashi M, Murata K, et al. (1992) Myocardial function and transmural blood flow during coronary venous retroperfusion in pigs. Circulation 86: 1265-1279.

28. Torry RJ, Myers JH, Adler AL, Liut CL, Gallagher KP (1991) Effects of nontransmural ischemia on inner and outer wall thickening in the canine left ventricle. Am Heart J 122: 1292-1299.

29. Reimer KA, Lowe JE, Rasmussen MM, Jennings RB (1977) The wavefron phenomenon of ischemic cell death. 1. Myocardial infarct size vs duration of coronary occlusion in dogs. Circulation 56: 786-794.

30. Vatner SF (1980) Correlation between acute reductions in myocardial blood flow and function in conscious dogs. Circ Res 47: 201-207.

31. Geer JC, Crago CA, Little WC, Gardner LL, Bishop SP (1980) Subendocardial ischemic myocardial lesions associated with severe coronary atherosclerosis. Am J Pathol 98: 663-680.
32. Stork A, Müllerleile K, Bansmann PM, Koops A, Meinertz T, et al. (2007) [Patterns of delayed-enhancement in MRI of ischemic and non-ischemic cardiomyopathies]. Rofo 179: 21-30.

33. Kroeker CA, Tyberg JV, Beyar R (1995) Effects of ischemia on left ventricular apex rotation. An experimental study in anesthetized dogs. Circulation 92: 3539-3548.

34. Götte MJ, Germans T, Rüssel IK, Zwanenburg JJ, Marcus JT, et al. (2006) Myocardial strain and torsion quantified by cardiovascular magnetic resonance tissue tagging: studies in normal and impaired left ventricular function. J Am Coll Cardiol 48: 2002-2011.

35. Henein MY, Gibson DG (1999) Long axis function in disease. Heart 81: 229231.

36. Ersbøll M, Valeur N, Mogensen UM, Andersen MJ, Møller JE, et al. (2012) Relationship between left ventricular longitudinal deformation and clinical heart failure during admission for acute myocardial infarction: a two-dimensional speckle-tracking study. J Am Soc Echocardiogr 25: 1280-1289.

37. Abate E, Hoogslag GE, Antoni ML, Nucifora G, Delgado V, et al. (2012) Value of three-dimensional speckle-tracking longitudinal strain for predicting improvement of left ventricular function after acute myocardial infarction. Am J Cardiol 110: 961-967.

38. Choi JO, Cho SW, Song YB, Cho SJ, Song BG, et al. (2009) Longitudinal $2 \mathrm{D}$ strain at rest predicts the presence of left main and three vessel coronary artery disease in patients without regional wall motion abnormality. Eur J Echocardiogr 10: 695-701.

39. Bijnens B, Claus P, Weidemann F, Strotmann J, Sutherland GR (2007) Investigating cardiac function using motion and deformation analysis in the setting of coronary artery disease. Circulation 116: 2453-2464

40. Urheim S, Edvardsen T, Torp H, Angelsen B, Smiseth OA (2000) Myocardial strain by Doppler echocardiography. Validation of a new method to quantify regional myocardial function. Circulation 102: 1158-1164.

41. Kramer CM, Rogers WJ, Theobald TM, Power TP, Petruolo S, et al. (1996) Remote noninfarcted region dysfunction soon after first anterior myocardial infarction. A magnetic resonance tagging study. Circulation 94: 660-666.

42. Götte MJ, van Rossum AC, Twisk JWR, Kuijer JPA, Marcus JT, et al. (2001) Quantification of regional contractile function after infarction: strain analysis superior to wall thickening analysis in discriminating infarct from remote myocardium. J Am Coll Cardiol 37: 808-817.

43. Jurcut R, Pappas CJ, Masci PG, Herbots L, Szulik M, et al. (2008) Detection of regional myocardial dysfunction in patients with acute myocardial infarction using velocity vector imaging. J Am Soc Echocardiogr 21: 879-886.

44. Berti M, Ghizzoni G, Gualeni A, Cantamessa P, Oneglia C (2012) Electrocardiographic pattern combined with echocardiographic wall motion abnormalities in stress related cardiomyopathies: clinical and pathophysiological insights. Arch Cardiol Mex 82: 22-30. 